\title{
ILCEA
}

Revue de l'Institut des langues et cultures

d'Europe, Amérique, Afrique, Asie et Australie

43 | 2021

Images des Amériques : fabrique, représentations, usages

\section{La représentation des Noirs dans le cinéma d'animation américain : entre pseudosciences et circulations intermédiatiques transnationales (1907-1946)}

Black Representation in American Animated Films: From Pseudoscientific

Depictions to Transnational Intermedial Circulations (1907-1975)

\section{Pierre Cras}

\section{OpenEdition}

\section{Journals}

Édition électronique

URL : https://journals.openedition.org/ilcea/13023

DOI : 10.4000/ilcea.13023

ISSN : 2101-0609

\section{Éditeur}

UGA Éditions/Université Grenoble Alpes

\section{Édition imprimée}

ISBN : 978-2-37747-299-4

ISSN : 1639-6073

\section{Référence électronique}

Pierre Cras, «La représentation des Noirs dans le cinéma d'animation américain : entre pseudosciences et circulations intermédiatiques transnationales (1907-1946) », ILCEA [En ligne], 43 | 2021, mis en ligne le 30 juin 2021, consulté le 30 juin 2021. URL : http://journals.openedition.org/ ilcea/13023 ; DOI : https://doi.org/10.4000/ilcea.13023

Ce document a été généré automatiquement le 30 juin 2021

(c) ILCEA 


\section{La représentation des Noirs dans le cinéma d'animation américain : entre pseudosciences et circulations intermédiatiques transnationales (1907-1946)}

Black Representation in American Animated Films: From Pseudoscientific Depictions to Transnational Intermedial Circulations (1907-1975)

Pierre Cras

\section{Introduction}

La présente réflexion vise à déconstruire les représentations des Noirs dans le cinéma d'animation américain et à infirmer l'idée selon laquelle ces dernières seraient purement figuratives et monolithiques. À travers l'analyse de stéréotypes essentialistes ${ }^{1}$ modelés par le racisme scientifique européen qui affirmait «scientifiquement " l'infériorité des « Noirs" face aux «Blancs», et repris à travers des transpositions caricaturales dans le cinéma d'animation ${ }^{2}$, cet article aborde le problème sous plusieurs angles. D'une part, nous reviendrons sur le discours raciste hérité des pseudosciences qui sous-tend la production d'images des Noirs dans le contexte de la première moitié du $\mathrm{xx}^{\mathrm{e}}$ siècle. D'autre part, nous tentons d'inscrire cette réflexion dans le cadre de l'analyse des représentations raciales ${ }^{3}$ aux États-Unis.

2 Nous avons sélectionné un panel de dessins animés produits par divers studios américains entre 1907 et 1946. Le cadre chronologique choisi s'étend de la première occurrence d'un archétype noir dépréciatif - le coon - dans le film d'animation américain Lightning Sketches (James Stuart Blackton, 1907) jusqu’à la sortie de John Henry and the Inky-Poo (George Pal, 1946) qui marquent un changement de paradigmes et les 
velléités réformatrices de l'industrie cinématographique de l'après Seconde Guerre mondiale. Nous traitons en priorité cette longue phase qui a défini esthétiquement et idéologiquement l'individu noir comme "Autre» dans le contexte américain par le truchement de la culture et de l'image animée.

Nous tentons d'explorer la complexité des représentations noires du cinéma d'animation américain à travers les procédés "d'altérisation ${ }^{4}$ " et de circulation intermédiatique ${ }^{5}$. Pour ce faire, nous aborderons d'abord l'influence de la discipline pseudoscientifique de la physiognomonie au sein des dessins animés américains lorsqu'il s'est agi de représenter des populations d'ascendance africaine, puis nous ferons de même avec la phrénologie avant de finalement nous pencher sur les évolutions au sortir de la Seconde Guerre mondiale.

Les exemples de dessins animés mobilisés lors de cette analyse se concentreront sur la période des années 1930 et 1940, soit l'âge d'or de l'animation américaine qui vit naître la majorité des productions étudiées.

\section{La physiognomonie, idéologie des passions}

\subsection{Physiognomonie et phrénologie, chantres de l'altérisation noire}

Qu'il s'agisse de la physiognomonie ${ }^{6}$, de la phrénologie ${ }^{7}$ ou des théories racistes de l'anthropologie physique, toutes ont en commun de consacrer "scientifiquement" l'idée selon laquelle les êtres vivants obéissent à un déterminisme biologique lié à l'appartenance à une même "race ». Comme le dit Guy Bechtel, l'une des idées maitresses était que tous les individus sont exclusivement dépendants de leur potentiel héréditaire. À cet égard, la «science » fournissait une justification à l'édification d'une hiérarchisation raciale mais aussi au sexisme et à l'existence de classes sociales. La corrélation entre aspect physique et valeur d'un individu atteint son paroxysme : «[...] tout inférieur dans la société était d'abord inférieur dans son corps. [...] Tous les gens du bas de l'échelle étaient des sous-produits [...]. » (Bechtel, 2002 : 29) La physiognomonie et la phrénologie partagent une volonté de théoriser les liens entre apparence extérieure et personnalité de l'individu, bien qu'elles mobilisent différentes méthodes selon le médecin moraliste Descuret en 1841 :

La physiognomonie et la phrénologie veulent toutes deux que notre extérieur ne soit que la manifestation de ce qui se passe habituellement au dedans de nous ; [...] ce principe admis, elles se séparent aussitôt, et procèdent d'une manière entièrement opposée : [...] l'une, reconnaissant le caractère par la configuration des traits qu'il a déterminés; l'autre, à la seule inspection des éminences cérébrales, traduites en relief sur le crâne, annonçant les instincts, les sentiments, les facultés qui prédominent et qui n'attendent que l'occasion favorable pour s'exercer. (1841: 119)

6 La physiognomonie, la phrénologie et le dessin animé - à travers la caricature de presse dont il est une extension ${ }^{8}-$ partagent un substrat idéologique commun: l'aspect externe d'un individu reflèterait ses inclinaisons, ses passions, ses faiblesses ${ }^{9}$. Tandis que la physiognomonie étudie les différentes inclinations et passions de l'homme, la phrénologie favorise l'étude des capacités et facultés propres à chacun. Les écrits de physiognomonie estiment que «sur le front habitent la lumière, la joie ; mais aussi le noir chagrin, l'angoisse, la stupidité, l'ignorance et la méchanceté». Autrement dit : « Le haut correspond à l'intellect, les passions abdominales se reflètent 
dans la partie basse, c'est-à-dire que le diable loge dans cette région. » (Lavater, $1841: 14)$

7 Ce clivage participe de la séparation entre les Européens au développement cérébral prononcé et les «autres" dont la proéminence maxillaire reflèterait l'inclinaison envers les domaines passionnels. Physiognomonie et phrénologie concluent que le visage des Noirs constitue autant de "preuves» de traits de caractères négatifs partagés par tous les Afro-descendants. Parallèlement à ces théories européennes se développent des idées voisines aux États-Unis inspirées par les travaux de Samuel George Morton, Josiah Clark Nott ${ }^{10}$ et George Gliddon. Adeptes de l'anthropologie et de la cranioscopie, ils affirment que la taille de la boîte crânienne reflète les capacités intellectuelles d'un individu et que l'espèce humaine provient de plusieurs souches différentes (polygénisme) selon l'interprétation racialiste de la variété des phénotypes humains. Dans leurs ouvrages Types of Mankind (1854) et Indigenous Races of the Earth (1857), Nott et Gliddon soutiennent que Blancs et Noirs appartiennent à deux races différentes et que leurs traits physiques n'ont jamais évolué au cours des siècles ; preuve de cette séparation.

8 Non contente de s'être exportée aux États-Unis au XIX ${ }^{e}$ siècle, la physiognomonie a connu une diffusion internationale dès le xviII ${ }^{\mathrm{e}}$ siècle et la publication des travaux de Johann Kaspar Lavater.

\subsection{Circulations transnationales de la physiognomonie}

9 Johann Kaspar Lavater, «fondateur » de la physiognomonie moderne, est né en 1740 à Zurich et est issu d'une famille de médecins. Il publie pour la première fois en 1775 et 1776 Physiognomische Fragmente zur Beförderung der Menschenkenntnis und Menschenliebe, soit La physiognomonie, ou l'art de connaitre les hommes d'après les traits de leur physionomie, leurs rapports avec les divers animaux, leurs penchants, etc., publié entre 1786 et 1803. Une première édition anglophone voit le jour en 1788. L'ouvrage rencontre une adhésion massive auprès des coreligionnaires protestants de Lavater qui détournent sa discipline à des fins d'altérisation racialiste et raciste ${ }^{11}$. Ainsi, dans $\mathrm{A}$ Brief Retrospect of the Eighteenth Century Containing a Sketch of the Revolutions and Improvements in Science, Arts, and Literature, During that Period, le révérend Samuel Miller livre une critique dithyrambique de l'ouvrage de physiognomonie de Lavater dont il confirme le succès international :

Non seulement en Suisse, mais également en Allemagne, en France, en Grande-Bretagne, et [...] à travers tout le monde littéraire, il a été lu avec un degré d'adoration et d'admiration rarement décernés aux productions d'un génie. Il a été traduit en différentes langues [...] et quoique sa popularité ait diminué, il est toujours utilisé avec un grand respect et plaisir. (1803:432).

Le Nouveau Monde s'empare du sujet avec exaltation. Entre la première édition anglaise des travaux de Lavater en 1788 et l'année 1826, 677 articles sont publiés dans des périodiques américains qui traitent de physiognomonie (Lukasik, 2011: 33). Nombre de ces travaux consacrent « scientifiquement » l'altérisation des Noirs. D'après l'Essai sur la physiognomonie des corps de 1797, l'Européen serait «blanc, sanguin; ses cheveux sont blonds, longs et touffus ; ses yeux sont ordinairement bleus ». À l'inverse, "l'Africain » serait «noir, phlegmatique [sic]; il a la fibre lâche ; ses cheveux sont très 
noirs, crépus ; sa peau est veloutée ; son nez est plat, ses lèvres sont grosses » (Sue, $1797: 58)$.

11 Ces assertions diffusées par la physiognomonie sur le physique des Noirs inchangé depuis des siècles se rapportent à des Africains, mais s'appliquent aussi aux AfricainsAméricains. Les exemples visuels qui reprennent cette uniformité sont légion dans la caricature de presse ${ }^{12}$ et dans le dessin animé aux États-Unis. De plus, s'il est bien un trait commun aux théories des zélateurs de la physiognomonie, c'est celui des appétits charnels et physiques des Noirs. Le pharmacien Julien-Joseph Virey ${ }^{13}$ défend en 1824 la prééminence des sensations physiques sur l'intellect parmi les populations noires (au contraire des Européens) :

Le nègre a les sens de l'odorat et du goût plus développés que le blanc [...] il sera donc plus adonné aux plaisirs des sens, non à ceux de l'esprit. Chez nous le front s'avance et la bouche semble se rapetisser, se reculer, comme si nous étions destinés à penser plutôt qu'à manger; chez le nègre le front se recule et la bouche s'avance, comme s'il était plutôt fait pour manger que pour réfléchir. (1824: 41).

Quant à la question du comportement social desdites populations, Virey affirme que les Noirs sont :

Rampants dans l'adversité, insolents dans la prospérité ; c'est une suite de l'esprit de servitude. Ils aiment aussi le faste, la dépense, le jeu, la bonne chère; ils recherchent surtout les vêtements les plus brillants, et poussent le luxe jusqu'à

l'extravagance quand la fortune les a émancipés. (Ibid. : 46).

13 Ces mêmes déclarations péremptoires et essentialistes inspireront directement les caricaturistes de presse américains qui se les réapproprient intégralement, bien que des années les séparent de la première diffusion de telles théories. En 1928, plus de 100 ans après la publication de l'ouvrage de Virey, le dessinateur de presse Eugene Zimmerman écrivait dans un manuel destiné aux caricaturistes et animateurs en herbe :

Les gens de couleur sont de bons sujets d'études [...]; ils sont des humoristes nés et adopteront souvent des attitudes ridicules ou prononceront des paroles hilarantes sans intention comique apparente. Le dessinateur joue habituellement sur l'amour que porte l'Homme de couleur aux vêtements criards, à la pastèque, au poulet, aux jeux de dés, et sur sa peur des fantômes, etc. (Matthews \& Zimmerman, $1928: 64$ )

En 1907 sortait aux États-Unis Lightning Sketches, le premier film d'animationattraction ${ }^{14}$ mettant en scène un personnage noir aux traits physiques volontairement exagérés: le coon. Le cadre de diffusion du film témoigne d'une dynamique intermédiatique ${ }^{15}$, combinant capture d'images filmées et performance scénique du protagoniste du film Stuart Blackton qui anime en «direct» la caricature de Noir née du terme péjoratif "coon». Selon Donald Bogle, le coon fut l'un des premiers archétypes de la culture américaine de masse à représenter la population noire sous un jour outrancier. Le coon était l'incarnation visuelle des assertions pseudoscientifiques susmentionnées: «Les coons [...] ont émergé comme des nègres bons à rien, indignes de confiance, mentalement dérangés, fainéants, ils étaient des créatures sous-humaines qui ne savaient que manger des pastèques, voler des poulets, jouer aux dés ou massacrer l'Anglais. » (Bogle, 1999 : 8)

15 S'il fait son entrée dans le cinéma d'animation en 1907, le coon est beaucoup plus ancien. On retrouve sa trace dans des productions culturelles du XIX ${ }^{e}$ siècle comme les comic strips, la caricature de presse ou les Minstrel Shows. Le coon de Lightning Sketches 
n'est pas une création ex nihilo de Stuart Blackton, mais le produit d'une circulation intermédiatique héritière indirecte des pseudosciences.

D'autres exemples de dessins animés participent de ce postulat stéréotypé qui voudrait que toutes les populations noires du monde entier soient identiques physiquement et culturellement.

\subsection{Uniformisation raciale et altérisation culturelle}

Dans le cinéma d'animation et de façon plus significative ${ }^{16}$ encore que dans le cinéma en prise de vues réelles, nombre de films reprennent l'idée d'une parenté biologique et d'une uniformité d'apparence entre populations noires "sauvages » et AfricainsAméricains. The Isle of Pingo Pongo (Tex Avery, 1938) est significatif à cet égard. Ce dernier est une parodie de documentaires ethnographiques qui prirent d'abord une forme littéraire avant d'être portés à l'écran au $\mathrm{xx}^{\mathrm{e}}$ siècle $^{17}$. La représentation des populations noires «primitives " que le film d'animation véhicule relève autant de la curiosité scientifique que des doctrines colonialistes occidentales. Ces dernières tendent vers la conclusion de l'infériorité de la «race nègre » et ont influencé moults représentations intermédiatiques en ce sens.

The Isle of Pingo Pongo retrace l'itinéraire d'un navire partant de New York jusqu'aux mers du Sud ${ }^{18}$. Une fois arrivé à destination, le narrateur décrit en voix off les habitants de l'île qu'il désigne comme "natives ${ }^{19}$. Leur apparence physique caricaturale à l'extrême rejoint les observations formulées par les disciplines du racisme scientifique. Ils ont des lèvres immenses, un front minuscule, des extrémités plates et larges, une peau foncée et se parent d'os dans les cheveux. Il est aussi impossible de distinguer les hommes des femmes autrement que par leurs vêtements.

Un autre aspect important de ce dessin animé réside dans sa mise en scène caricaturale d'une similitude physique et culturelle entre les Noirs du monde entier que la physiognomonie résumait au XIX ${ }^{\mathrm{e}}$ siècle :

La compression du crâne, le nez épaté, le museau avancé, les lèvres épaisses, les dents incisives obliques, les cheveux crépus et laineux. (Delestre, 1866 : 88)

Si l'on compare l'ensemble de la race noire à celui des autres familles, l'on constatera moins de divergences dans l'expression graphique des noirs entre eux. (Ibid. : 96-98).

Dans l'une des séquences du film, alors que les indigènes de l'île sont regroupés en couples de danseurs, le narrateur annonce : « Filling the air with a pandemonium of vibrant jungle rythm, a native celebration gets under way ${ }^{20}$. " Le spectateur s'attend à assister à une cérémonie traditionnelle, mais les locaux entonnent Sweet Georgia Brown, une chanson de jazz américain enregistrée le 13 mai 1925 (Sullivan, 2013 : 590) et rendue célèbre par son interprétation par la chanteuse Ethel Waters, reprise ensuite comme hymne par l'équipe de basketball des Harlem Globetrotters (Buchanan, 2005: 44). Du fait de sa popularisation par des Africains-Américains influents, Sweet Georgia Brown est ancrée dans l'imaginaire collectif américain. Le choix de cette chanson pour illustrer la « fête autochtone" est porteur de sens et consacre la théorie pseudoscientifique d'une uniformité à la fois physionomique et culturelle des peuples noirs.

21 Comme pour étayer cette hypothèse, la séquence suivante dévoile au spectateur les interprètes de Sweet Georgia Brown. Ce sont quatre natifs vêtus de pagnes, de nœuds papillons et qui se livrent à une parodie de spectacle musical. Le cœur formé par les 
quatre chanteurs est une caricature des Mills Brothers, un célèbre quatuor noir américain fondé en 1928 (Goldmark, 2005 : 92). La confusion volontairement opérée par Tex Avery contribue à associer dans l'imaginaire collectif l'archétype du sauvage, du jazzman africain-américain et de la caricature ponctuelle d'artistes noirs célèbres. L'utilisation particulière d'éléments relevant du racisme "scientifique ", de la culture populaire américaine et de la caricature de presse place The Isle of Pingo Pongo dans la catégorie des œuvres cinématographiques qui confirment notre postulat relatif à l'hybridité et l'intermédialité du film d'animation.

Si les considérations esthétiques et idéologiques héritées de la physiognomonie puis de la caricature de presse sont réutilisées dans le dessin animé américain inscrivent de telles représentations dépréciatives des Noirs dans la psyché américaine, il en va de même pour la phrénologie.

\section{Transpositions intermédiatiques de la phrénologie}

\subsection{Une pseudoscience essentialisante et politisée}

Lorsqu'il publie son ouvrage Phrenology, Or the Doctrine of the Mind en 1825, Johann Gaspar Spurzheim met en place les principes de la phrénologie moderne. Il délaisse l'idée d'un cerveau comme uniforme et divise celui-ci en « organes » qui possèdent une topographie fixe et précise à l'intérieur de la boîte crânienne. Chacun de ces organes est associé à des capacités et traits de caractère particuliers classés en deux catégories. La première concerne "l'affectif » et regroupe les "propensions » (combativité, esprit de construction et de destruction...) et les "sentiments" (estime de soi, vénération, fermeté...). La deuxième catégorie concerne "l'intellect» et regroupe les " perceptions » (capacité à calculer, à créer des repères temporels, à communiquer...) ainsi que les capacités de "réflexion » (développer la notion de causalité). Spurzheim introduit auprès du grand public cette discipline qu'il présente comme « la doctrine des facultés particulières de l'esprit, et des relations entre leurs manifestations et celles du corps ; tout particulièrement celles du cerveau » $(1825: 1)$.

À partir des années 1830, la phrénologie se diffuse aux États-Unis. Dans son journal de bord, la sociologue Harriet Martineau décrit le succès rencontré par Spurzheim lors de sa visite en Nouvelle-Angleterre en 1832. La "majorité de la société est devenue phrénologue en une journée" après les interventions de Spurzheim (Martineau, 1838:188; McCandless, 1992: 199). En quelques années, l'Europe et les États-Unis adhèrent largement aux théories d'altérisation raciales de cette nouvelle "science ". Dès les années 1840 , la diffusion dans le monde anglophone se poursuit via des "Sociétés phrénologiques " comme la Boston Phrenological Society, fondée en 1832 (Bartlett, 1838:148) ou de journaux tels The Annals of Phrenology créé en 1833 ou The American Phrenological Journal and Miscellany créé en 1836.

Dès ses débuts, la phrénologie est politisée. Lorsque le phrénologue écossais George Combe effectue un phrenological tour à partir du 18 octobre $1838^{21}$, il revient sur la façon dont la phrénologie doit servir les intérêts de l'Amérique. Dans son discours intitulé Notes on the United States of North America, during a Phrenological Visit in 1838-39-40, Combe admire le caractère national américain. Il loue «l'esprit d'estime de soi qui conduisit vos ancêtres sur ces rivages [...] et vous a permis d'accomplir votre indépendance », les institutions américaines qui « incitent fortement l'homme public à délaisser la flatterie 
pour la vérité » ou encore le courage moral du peuple qui le guide vers «la recherche de la vérité et du pragmatisme au mépris de tous les dangers » (1839:362). George Combe s'inscrit ici dans un courant d'idéologie politique américain qu'Arthur Wrobel identifie comme celui de l'idéal de «l'optimisme jacksonien", d'après le nom de l'ancien président Andrew Jackson (Wrobel, 1987 : 123).

Il n'y a rien d'étonnant à ce que la phrénologie ait été accueillie favorablement au cours de cette période particulière qui faisait suite à l'ère des «bons sentiments ». Pendant cette dernière, les États-Unis aspiraient à l'unité autour d'un but national malgré les divisions entre Sud et Nord. Dès l'avènement de la démocratie jacksonienne en 1825, l'esprit d'union nationale n'est plus et l'altérisation bat son plein. Les Amérindiens virent ainsi toute leur organisation sociale bouleversée dès 1830 par l'Indian Removal $A c t^{22}$ et firent l'objet d'une " détribalisation » à but d'acculturation. L'altérisation des Noirs était pour sa part déjà en place dans le système constitutionnel ; la mise au ban de leur humanité et de leur citoyenneté avait été entérinées dès 1787 par l'adoption du compromis des $3 / 5^{\mathrm{e}}$. L'identité américaine comprenait une définition politique et sociale de la norme qui se manifestait aussi par la culture - via les caricatures de la presse satirique, puis dessins animés ${ }^{23}$ - et constituait de facto un terreau fertile pour la réception de la phrénologie et de ses théories racistes.

\subsection{De la phrénologie au dessin animé}

Dans le cadre de l'approche intermédiatique de cette réflexion, il est révélateur de se pencher sur l'importance que la phrénologie - et dans une moindre mesure la physiognomonie - accordent à l'enveloppe extérieure qui protège les organes cérébraux : « Le cerveau est logé sous une enveloppe solide et résistante. [...] Cette boîte osseuse a reçu le nom de crâne ; c'est le coffre-fort des facultés. » (Delestre, 1866 : 159) Selon la phrénologie, la forme du crâne serait déterminante dans la répartition des facultés et reflèterait les inclinations et les capacités de chacun. Chaque irrégularité de la boîte crânienne indiquerait un manque, un excès ou une singularité des régions cérébrales correspondantes. Lorsque les auteurs du Dictionnaire d'histoire naturelle appliquée aux arts indiquent en 1803 que « le crâne des nègres est communément plus étroit que celui des Européens ", ils établissent l'infériorité de développement des organes cérébraux noirs face à ceux des Européens ${ }^{24}$. Le crâne déprécié serait une conséquence physique de cette inégalité des facultés intellectuelles. Une observation pseudoscientifique qui trouva un écho renouvelé dans le contexte de la crise économique des années 1930 qui vit un repli isolationniste s'opérer aux États-Unis et s'accompagner d'un rejet de l'altérité.

Bien que la phrénologie ne soit plus considérée comme une science lors de l'âge d'or du cinéma d'animation, les stéréotypes auxquels elle avait donné naissance étaient présents dans l'imaginaire collectif américain. De nombreux dessins animés mettaient en image les théories de la phrénologie sur la forme et à la taille du crâne des Noirs. L'un d'entre eux, All This and Rabbit Stew, transpose visuellement la corrélation entre l'aspect de la boîte crânienne, les capacités intellectuelles et les inclinaisons plus ou moins marquées d'un individu. Ce dessin animé de 1941 fait partie de la série des Bugs Bunny ${ }^{25}$ (Shull \& Wilt, 2004: 211) et voit ce dernier être pourchassé par un individu armé qui se fait avoir par les astuces et tours savamment exécutés du lapin. Le chasseur Elmer Fudd est ici remplacé par un personnage noir qui reprend la voix aigrelette et la 
démarche dégingandée de l'acteur Stepin Fetchit, incarnation vivante de l'archétype du coon.

L'archétype du coon usité par Tex Avery dans ce film regroupe tous les traits physiques et comportementaux maintes fois raillés et déconsidérés par les cartoonistes. Il rejoint les observations de l'ouvrage Histoire naturelle du genre humain en $1824:$ « [...] les nègres à front petit, aux yeux enfoncés, à regard faux [...] passent ordinairement pour les plus redoutables par leur mauvais caractère.» (Virey, $1824: 160$ ) En effet, le personnage du coon de Tex Avery fait non seulement preuve d'un manque d'entrain et d'intellect mais il est pusillanime et obstiné comme en témoignent toutes les humiliations qu'il essuie sans discontinuer pendant sept minutes. All This and Rabbit Stew se clôt par une séquence qui confirme le statut de "sucker » de cette caricature et durant laquelle Bugs Bunny et ce dernier jouent aux dés afin de mettre un terme à leur différend. Le chasseur noir archétypal perd la partie, ses vêtements, son fusil, et se retrouve nu face à un Bugs Bunny triomphant.

\subsection{Le dessin animé américain héritier de la « Cause perdue »?}

30 La superposition des traits physiques peu avantageux décrits plus haut et d'une attitude stéréotypée inscrivent All This and Rabbit Stew dans la continuité des traités phrénologiques et physiognomoniques. Le personnage rejoint les dires du phrénologue Castle quant au «tempérament bilieux », caractérisé par une prédominance du système veineux, musculaire et osseux chez les Noirs :

La chevelure est noire et mate ; les lèvres d'un rouge bleuâtre, les yeux d'un brun jaune [...] le pouls lent, irrégulier et mou ; l'individu éprouve de la répugnance pour tout ce qui est mouvement; l'activité intellectuelle amène promptement de la fatigue et de fréquents accès de sommeil. $(1862: 207)$

31 Cependant, le dessin animé américain qui illustre peut-être le plus ouvertement cette théorie de l'apathie des Noirs joint à l'esthétique caricaturale un fond dépréciatif. Il s'agit de Scrub Me Mama with a Boogie Beat (Walter Lantz, 1941). La scène d'exposition situe l'action dans un lieu fictif inspiré par du Sud rural des États-Unis postReconstruction. Les champs de coton, bateaux à aubes et maisons de bois défilent au rythme de la chanson old Folks at Home ${ }^{26}$. La caméra s'arrête sur un habitant de la ville de "Lazy Town » dans laquelle se déroule l'action. Le personnage apparaît dans les notes de production de Walter Lantz sous le nom de "nigger fishing " et possède des traits caricaturaux quasi simiesques ${ }^{27}$. Il est allongé sur un lit flottant et est trop paresseux pour se laver comme l'indique la présence des mouches autour de lui.

Tous les habitants de Lazy Town sont décrits dans les notes de production internes au studio Warner comme des "fainéants » incapables de pêcher ou de réagir à des piqûres de moustiques. Alors qu'une bagarre d'une mollesse incroyable éclate entre deux Noirs paresseux, un bateau à aube accoste. Une jeune femme noire élégante à la peau claire répondant au nom de Nellie (de Harlem) - la mention de son lieu d'origine en fait une citadine sophistiquée ${ }^{28}$ - en descend et suscite immédiatement l'intérêt/l'excitation de tous les hommes de la ville qui se réveillent en sursaut ${ }^{29}$. Venue rendre visite à sa mère alors en pleine lessive, elle lui donne le conseil suivant: «Écoute, maman. C'est pas une façon de laver des vêtements. T'as besoin de RYTHME $^{30}$ !» Nellie entonne alors la chanson-titre Scrub Me Mama with a Boogie Beat et 
entraîne toute la population de Lazy Town qui se déhanche au rythme de la bande sonore du film.

Les personnages du film sont donc fainéants, sales, pauvres, mais se complaisent dans leur indigence et ne se soucient guère de leur situation. Cette incapacité à faire face à l'avenir se retrouve dans les écrits de physiognomonie et phrénologie lorsque Julien-Joseph Virey annonce que "l'Africain pousse encore plus loin l'apathie et l'imprévoyance de l'avenir. Les vaisseaux négriers qui font la traite des esclaves ont toujours quelques musiciens à bord, pour faire oublier aux nègres toute la misère de leur état" (1824:48). Scrub Me Mama with a Boogie Beat reprend littéralement cette observation, l'applique au film l'animation et l'adapte au contexte du Sud des États-Unis. Le dessin animé se saisit de la relation fusionnelle du corps noir avec la musique, seul élément capable de sortir les Noirs de leur apathie et renforce le stéréotype du « rythme dans la peau » prégnant dans l'imaginaire collectif ${ }^{31}$.

La représentation caricaturale et raciste du Sud de l'après-guerre de Sécession est aussi en jeu. Par son imagerie qui oscille entre révisionnisme romantique et stigmatisation des populations noires incapables, le dessin animé rejoint le mythe révisionniste de la "Cause perdue ». Ce dernier considère que l'esclavage ne joua qu'un rôle mineur dans les événements déclencheurs de la guerre de Sécession (1861-1865), que la Reconstruction (1863-1877) vit la destruction de la culture traditionnelle sudiste par l'Union et que l'esclavage était une institution bienveillante au sein de laquelle les esclaves noirs étaient heureux (Taylor, 2002: 266). Certaines productions cinématographiques américaines telles que Naissance d'une nation (D. W. Griffith, 1915) font montre d'une continuité idéologique avec le mythe de la "Cause perdue ». Il en va de même pour le cinéma d'animation des années 1930 et 1940, à plus forte raison lorsque les réalisateurs représentent un Sud rural laissé à l'abandon par des Noirs indolents régis par un pseudo déterminisme biologique.

Avec la fin de la Seconde Guerre mondiale et les revendications des vétérans noirs revenus d'un front européen où la ségrégation de jure n'existait pas dans l'espace public, les schémas de représentations racistes de l'animation évoluent. L'un des témoins de cette évolution se nomme George Pal.

\section{4. À l'Est, du nouveau : le cas George Pal}

\subsection{Little Jasper et son évolution}

L'une des utilisations notables d'archétypes noirs anciens est celle du réalisateur George Pal à l'occasion de sa série Little Jasper dont les aventures sont relatées à travers 15 courts-métrages produits entre 1942 et 1946 (Hickman, 1977:22; Holman, 1975 : 104). Jasper est un petit garçon noir du Sud qui vit en compagnie de personnages divers comme un cheval capricieux ou un corbeau moqueur. Les premières années d'existence de Jasper sont marquées par la reprise d'éléments issus du Blackface Minstrelsy et du dessin animé Little Black Sambo (1935). Jasper vit avec sa mammy dans une cabane et évolue dans un environnement rural. Il s'exprime avec un " parler noir » vernaculaire caricatural du Sud, est peureux et rêve d'une contrée où les pastèques sont aussi grandes que des maisons.

Il existe une différence notable entre les intentions premières du cinéaste George Pal et le résultat final. Selon Sondra Gorney, le personnage de Little Jasper tel qu'il est visible 
par exemple dans Jasper and the Watermelons est un "stéréotype noir que les Noirs eux-mêmes réprouvent » (1946: 372). Cependant, ce n'était pas la volonté de George Pal que de créer un personnage qui réponde à l'imagerie dépréciative dominante. Il disait avoir un profond respect pour le folklore noir qu'il estimait être le plus riche de l'histoire des États-Unis (ibid. : 373)

La fascination de George Pal pour la culture noire américaine s'explique en partie par sa vision idéalisée de l'Amérique. Né en Hongrie en $1908^{32}$ dans une famille d'artistes, il obtint un diplôme de l'Académie des arts de Budapest à l'issue de sa scolarité. Il exerçait le métier de dessinateur de presse et fut remarqué pour ses capacités artistiques puis créa son propre studio en Hollande. Pal fut ensuite approché par les studios Paramount en 1939 (Solomon, 1987:8) qui lui proposèrent de continuer à produire sa série-phare: George Pal's Puppetoons. Il quitta alors la Hollande avec sa famille pour vivre le rêve américain ${ }^{33}$.

L'expérience américaine de George Pal et sa vision de la population noire sur place sont perçues au travers d'un prisme différent de celui des animateurs nés aux États-Unis. Lorsqu'il réalise le premier épisode de la série Little Jasper, George Pal n'est pas au fait de la situation des Noirs dans le pays car il n'a pas croisé d'animateurs noirs dans les studios $^{34}$. Une ingénuité évoquée par les articles de la presse noire qui critiquent ouvertement le recours aux caricatures de la série Little Jasper :

En tant qu'Européen qui n'a pas été élevé dans les préjugés racistes, il considère l'Amérique sous l'angle de ce qu'il y trouve. Pour lui, il n'y a rien de déplacé à représenter un petit garçon noir qui aime manger des pastèques ou qui est effrayé lorsqu'il traverse une maison hantée. Mais pour les Noirs américains qui tentent de se débarrasser du mythe Oncle-Tomesque des Noirs puérils qui ne mangent rien d'autre que de la mélasse, des pastèques, et ont peur de leur ombre, Jasper est désagréable. (Ebony, Cohen, 1997 : 58)

En tant qu'émigré européen aux États-Unis le réalisateur n'a eu affaire avant 1938 qu'à des représentations noires façonnées par le processus d'altérisation et qui relèvent de la caricature. Eu égard à la diffusion internationale de la vision nostalgique du Sud esclavagiste à travers Minstrel Shows ou films divers, il ne serait pas surprenant que cette imagerie ait inspiré Little Jasper. George Pal fait évoluer ses personnages : il modifie les caractéristiques de Jasper au fil de ses péripéties et lui ôte son parler noir caricatural à partir de Jasper's Derby en 1946.

\subsection{John Henry, fer de lance d'un nouveau contre-discours}

41 En cette même année 1946, Pal met en scène son premier personnage noir héroïque qui n'est ni humoristique ni satirique. John Henry and the Inky Poo est né de cette volonté du cinéaste de réaliser un film hommage aux contes africains-américains. Ce courtmétrage reprend un récit traditionnel du combat entre John Henry et une locomotive qui s'affrontent lors d'une course. L'issue de celle-ci déterminera si l'humain est capable de triompher de la machine. Après avoir remporté l'épreuve au prix d'un effort surhumain, John Henry s'effondre et meurt d'épuisement.

42 À l'inverse des représentations archétypales utilisées dans des films d'animation de la période, John Henry n'est pas un personnage aux proportions caricaturales héritées des principes pseudoscientifiques, mais un homme fort comme en atteste sa description dans le conte originel : « John Henry était grand. John Henry était fort. Il mesurait deux mètres de large et trois mètres de haut. » (Gorney, 1946 : 373) On note également que le 
film fait montre d'une certaine volonté d'authenticité culturelle puisqu'il choisit de ne pas faire appel à un acteur blanc qui imiterait un Noir comme c'était le cas dans les exemples de dessins animés étudiés précédemment ${ }^{35}$. Le narrateur présent en voix off pendant toute la durée du film est ainsi incarné par l'acteur noir Rex Ingram dont la carrière florissante sur le grand écran est à l'époque appréciée du public noir américain (Boyd, 2008 : 97). L'initiative de George Pal est importante et fait écho à la carrière de l'acteur qui s'est toujours efforcé d'incarner des personnages positifs qui « échappaient à la typologie rigide à laquelle la plupart des acteurs noirs étaient confrontés » (Bogle, 1999 : 70).

\section{Conclusion}

La période de l'après-guerre est marquée par une certaine ambiguïté ; la représentation des Africains-Américains dans le dessin animé évolue après 1946, mais la présence de personnages noirs archétypaux archaïques est encore vivace jusqu'aux années 1950.

D'un côté, de nouvelles images progressistes des Noirs se diffusent à travers certains films d'animation novateurs en la matière. On peut par exemple citer le plaidoyer antiraciste Brotherhood of Man (1946) qui déconstruit avec pédagogie les idées racistes et pseudoscientifiques d'inégalité biologique entre les « races » humaines qui prévalaient jusqu'à lorsqu'il s'agissait de représenter des Noirs. La découverte des camps nazis par les soldats américains et l'idéologie raciste mortifère qui leur étaient associés n'est probablement pas étrangère à ce revirement des studios d'animation.

Mais d'un autre côté, cette même période témoignera - au moins jusqu'à la deuxième moitié des années 1950 - d'une survivance de certains archétypes anciens comme la mammy dont la présence montre la difficile accession à un changement des mentalités malgré des velléités progressistes. À cet égard, les réactions contradictoires entre admiration et indignation suscitées par la sortie du film de Walt Disney Mélodie du Sud en 1946 portent en elle la marque de cette période de transition qui fut déterminante pour la suite.

\section{BIBLIOGRAPHIE}

BARRIER Michael (1999), Hollywood Cartoons. American Animation in Its Golden Age, New York : Oxford University Press.

BARTLETT Elisha (1838), « An Address, Delivered at the Anniversary Celebration of the Birth of Spurzheim and of the Organisation of the Boston Phrenological Society, January 1, 1838 ", The American Phrenological Journal and Miscellany, I.

BeCHTEL Guy (2002), Délires racistes et savants fous, Paris : Plon.

BoGLE Donald (1999), Toms, Coons, Mulattoes, Mammies \& Bucks: An Interpretive History of Blacks in American Films, New York : Continuum. 
BoYD Todd (2008), African Americans and Popular Culture, Westpert : Praeger.

BUCHANAN Paul D. (2005), Race in the United States: A Chronology, 1896-2005, Jefferson : McFarland.

CASTLE Michel-Arthur (1862), Phrénologie spiritualiste : nouvelles études de physiologie appliquée, Paris : Didier \& C

COHEN Karl F. (1997), Forbidden Animation. Censored Cartoons and Blacklisted Animators in America, Jefferson : McFarland.

COQUET-Мококо Cécile (2018), Mémoire de synthèse. Candidature à une Habilitation à diriger des recherches, Paris : Université Paris 3 Sorbonne-Nouvelle.

COMBE George (1839), Lectures on Phrenology, New York : Samuel Colman.

CRAFTON Donald (1993), Before Mickey: The Animated Film, 1898-1928, Chicago : The University of Chicago Press.

Delestre Jean-Baptiste (1866), De la physiognomonie : texte, dessin, gravure, Paris : Jules Rénouard.

DESCURET Jean-Baptiste Félix (1841), La médecine des passions, ou les passions considérées dans leurs rapports avec les maladies, les lois et la religion, Paris : Béchet et Labé.

GAUDREAULT André (2008). Cinéma et attraction : pour une nouvelle histoire du cinématographe, Paris : CNRS Éditions.

Goldmark Daniel Ira (2005), Tune for 'Toons: Music and the Hollywood Cartoon, Los Angeles :

University of California Press.

GoRnEY Sondra (1946), « The Puppet and the Moppet », Hollywood Quarterly, 1(4), 371-375.

HICKMAN Gail Morgan (1977), The Films of George Pal, New York : Yoseloff.

Holman L. Bruce (1975), Puppet Animation in the Cinema: History and Technique, New York : Barnes. JUTEAu Danielle (2006), « Forbidding Ethnicities in French Sociological Thought: The Difficult Circulation of Knowledge and Ideas », Mobilities, 1(4), 399-400.

LAVATER Johan Kaspar (1841), La physiognomonie, ou l'art de connaître les hommes d'après les traits de leur physionomie, leurs rapports avec les divers animaux, leurs penchants, etc., Paris : Librairie française et étrangère.

LE RouSSEAU Julien (1847), Notions de phrénologie, Paris : Librairie phalanstérienne.

LUKASIC Christopher J. (2011), Discerning Characters: The Culture of Appearance in Early America, Philadelphie : University of Pennsylvania Press.

MARTINEAU Harriet (1838), Retrospect of Western Travel, Londres : Saunders and Otley.

MATthews E. C. \& Zimmerman Eugene (dit Zim) (1928), How to Draw Funny Pictures: A Complete Course in Cartooning, Chicago : Frederick J. Drake \& Co.

MCCANDLESS Peter (1992), « Mesmerism and Phrenology in Antebellum Charleston: "Enough of the Marvellous" ", The Journal of Southern History, 58(2), 199-230.

MiLlER Samuel (1803), A Brief Retrospect of the Eighteenth Century: Part First (vol. 1), New York : J. Swords.

PARFAIT Claire (2007), The Publishing History of Uncle Tom's Cabin, 1852-2002, Hampshire : Ashgate. PIETERSE Jan Nederveen (1992), White on Black: Images of Africa and Blacks in Western Popular Culture, New Heaven : Yale University Press. 
SPURZHEIM Johann Gaspar (1825), Phrenology, Or the Doctrine of the Mind: And of the Relations Between Its Manifestations and the Body, Londres : William Clowes.

STACK, Richard A. (2006), Dead Wrong: Violence, Vengeance, and the Victims of Capital Punishment, Westport : Praeger.

ShULl Michael \& WiLt David (2004), Doing Their Bit: Wartime American Animated Short Films, 1939-1945 ( $2^{\mathrm{e}}$ éd.), Jefferson : McFarland.

SUE Jean-Joseph (1797), Essai sur la physiognomonie des corps vivans considérée depuis l'Homme jusqu'à la plante, Paris : L'Auteur, Dupont.

SulLIVAN Steve (2013), Encyclopedia of Great Popular Song Recordings (vol. 1), Londres : Scarecrow Press.

TAYLOR Helen (2002), « Gone With the Wind and Its Influence », C. Perry \& M. Weaks-Baxter (éds), The History of Southern Women's Literature, Baton Rouge : Louisiana State University Press.

VIREY Julien-Joseph (1824), Histoire naturelle du genre humain, nouvelle édition entièrement refondue ; avec figures (vol. 2), Paris : Crochard.

WROBEL Arthur (1987), Pseudo-Science and Society in 19th Century America, Lexington : University Press of Kentucky.

\section{NOTES}

1. Axés majoritairement autour $\mathrm{du}$ manque d'intelligence et de civilisation des populations d'ascendance africaine au profit de leur corporéité (vigueur sexuelle et athlétique comprises) et de leurs appétits charnels variés.

2. Nombre d'artistes des premiers studios d'animation ont été caricaturistes de presse et ont transposé les pratiques du dessin satirique (exagération des traits physiques et des proportions, déformation des corps, volonté de tourner en ridicule des protagonistes/situations, recours à des stéréotypes afin de capter immédiatement l'attention du lecteur/spectateur, etc.) à l'animation en y ajoutant le mouvement et le langage oral à partir du premier dessin animé parlant en 1928.

3. Nous préférerons utiliser ce terme plutôt que celui, composite, d'« ethno-racial » afin de ne pas tomber dans le "nationalisme méthodologique empêchant l'analyse de dynamiques ethniques " évoqué par la sociologue québécoise Danielle Juteau. Comme le disent par Jan Nederveen Pieterse, Pierre-Jean Simon ou Cécile Coquet-Mokoko, le concept social de « race » a connu un glissement sémantique à partir de la deuxième moitié du $\mathrm{XIX}^{\mathrm{e}}$ siècle pour ajouter aux considérations phénotypiques une composante culturelle.

4. Défini par Henri Courau dans son ouvrage Ethnologie de la Forme-camp de Sangatte : de l'exception à la régulation: "J'entends par "altérisation" la contraction de deux termes, l'un renvoyant au phénomène de la production d'altérité, l'autre à l'altération. L'«altérisation » induit donc un mouvement simultané de production de l'altérité s'effectuant dans la soustraction ou dans la mise en abyme d'un élément d'identification initialement employé. »

5. L'adjectif « intermédiatique » renvoie à la notion « d'intermédialité » développée par « l'école de Montréal " et les chercheurs regroupés depuis 1997 au sein du Centre de recherche sur l'intermédialité (CRI) devenu en 2010 le Centre de recherches intermédiales sur les arts, les lettres et les techniques (CRIalt). Le concept d'intermédialité comprend ici les interactions entre différents médias regroupés autour d'un objet et d'une série culturelle commune : le continuum esthétique et idéologique dépréciatif des individus africains ou d'ascendance africaine. 
6. Discipline pseudoscientifique qui vise à connaître le caractère et les inclinations d'un individu d'après la conformation des traits de son visage.

7. Soit l'étude pseudoscientifique des capacités et du caractère d'un individu d'après l'analyse de la forme de sa boîte crânienne.

8. Nous utilisons l'idée défendue par Isabel Simeral Johnson dans «Cartoons » publié en 1937 dans The Public Opinion Quarterly, p. 21. Cette proximité entre les deux formes d'art s'exprime à travers la polysémie du terme « cartoon » qui recouvre le dessin satirique (particulièrement celui des comic strips) et le dessin animé.

9. Voir Laurent Baridon \& Martial Guédron, Corps et arts : physionomies et physiologie dans les arts visuels, Paris : L'Harmattan, 1999.

10. Originaire de Caroline du Sud, il a vécu la majeure partie de sa vie en Alabama. L'imaginaire collectif sudiste raciste et esclavagiste dont il était familier a sûrement influencé ses travaux. Les théories médicales et pseudoscientifiques européennes ont aussi orienté ses recherches en anthropologie physique et craniométrie après qu'il eût visité l'Europe en 1835 .

11. Bien que Lavater ait constamment inscrit ses recherches sous le regard de Dieu (l'être humain a été créé à son image et porte donc une part de divinité), il devient beaucoup plus compliqué de quantifier l'influence exacte de la religion dans les écrits ultérieurs des partisans de la physiognomonie et des pseudosciences.

12. En particulier à travers Puck, Life et Judge, les trois journaux satiriques les plus populaires du XIX ${ }^{\mathrm{e}}$ siècle aux États-Unis.

13. Sa théorie racialiste a inspiré Josiah Clark Nott pour son ouvrage Two Lectures on the Natural History of the Caucasian and Negro Race (1844).

14. Le terme "cinéma-attraction" renvoie aux pratiques du médium avant son institutionnalisation vers 1913. L'usage de cette notion permet de ne pas rompre le continuum entre des formes antérieures d'arts scéniques (vaudeville, cirque, théâtre) et le cinématographe contrairement à celle de « cinéma des premiers temps ».

15. Voir Gaudreault (2008).

16. En particulier à cause des possibilités graphiques offertes par le medium, qui peut recréer à l'envi et sans les contraintes physiques inhérentes au cinéma en prise de vues réelles, les stéréotypes les plus extrêmes par l'entremise de la déformation des corps des personnages.

17. Voir Tim Youngs, Travellers in Africa: British Travelogues, 1850-1900, Manchester University Press, 1994.

18. L'itinéraire et la destination finale du navire ne sont pas explicitement mentionnés, bien que le spectateur comprenne que c'est une version fantasmée et exotique de la "jungle africaine » qui est mise en scène ici.

19. "This Isle of Pingo Pongo (1938)", fonds Warner Bros. Archives, Merrie Melodie No. 8402, carton numéro 1114, University of Southern California.

20. Warner Bros Archives, USC, ibid.

21. Qu'il relate dans le chapitre «The Application of Phrenology to the Present and Prospective Condition of the United States ", Lectures on Phrenology (1839).

22. Proposée par Andrew Jackson, votée par le Congrès puis signée par le Président en avril 1830, l'Indian Removal Act mit en place la déportation forcée des Amérindiens qui vivaient sur le territoire situé à l'est du Mississippi vers les territoires « inoccupés » de l'Ouest.

23. Quand bien même le dessin animé fut grandement expurgé de l'aspect politique propre à la presse satirique.

24. Société de naturalistes et d'agriculteurs, Nouveau dictionnaire d'histoire naturelle appliquée aux arts, principalement à l'agriculture et à l'économie rurale et domestique, Paris, Imprimerie de Crapelet, 1803, p. 114. 
25. Personnage aperçu pour la première fois en 1938 dans Porky's Hare Hunt, Bugs Bunny est l'incarnation animée de la figure du trickster qui utilise la ruse, la diversion ou la tergiversation afin de triompher d'une situation défavorable ou d'un ennemi plus puissant que lui.

26. « Notes de production », Walter Lantz Archive 047, Productions for Theatrical Release, Scrub Me Mama with a Boogie Beat, dossier 26, Folder 3, University of California Los Angeles.

27. Pour en savoir plus sur la simianisation des personnages noirs de cartoon, voir Pierre Cras, «Simianisation, fantasmagorie et imaginaire racistes dans le cartoon aux États-Unis ", Revue de la $B N F, n^{\circ} 61,2020$, p. 85-95.

28. C'est une « élégante » de Harlem.

29. «Dialogue sheet. Prod. \#999», Walter Lantz Archive 047, Productions for Theatrical Release, Scrub Me Mama with a Boogie Beat, dossier 26, Folder 3, UCLA.

30. Ibid.

31. Lorsque Scrub Me Mama with a Boogie Beat ressort sur les écrans américains en 1948, il suscite de vives réactions de la part de la National Association for the Advancement of Colored People (NAACP). Deux représentants de cette association qui œuvre pour les droits des Noirs américains depuis 1910 s'insurgent du fait que les personnages sont fainéants et ne se meuvent qu'en musique.

32. Charles Solomon, "Movie Reviews: Paying Homage to Pal's "Puppetoons" ", coupure de presse d'un journal inconnu, vendredi 12 juin 1987, p. 8, George Pal Collection, Puppetoons, carton numéro 2, dossier numéro 4, "George Pal Papers 1937-1986», collections spéciales, University of California Los Angeles, cote : PASC 110.

33. Annie Awards Brochure, 1976, p. 3, Walter Lantz Archive 047, archives personnelles, carton numéro 74, dossier numéro 12, collections spéciales, University of California Los Angeles, cote : PASC 47.

34. Frank Braxton, le premier animateur africain-américain n'a été recruté qu'en 1950, soit huit ans après le premier épisode de la série des Little Jasper.

35. Bien qu'aucune culture ne soit monolithique dans son essence et que la mondialisation sous toutes ses formes ait chamboulé la notion même d'authenticité et de tradition, nous faisons ici référence à la culture qui émane d'artistes africains-américains et non la mise en scène d'une imitation voire la parodie de celle-ci par des animateurs blancs.

\section{RÉSUMÉS}

Depuis l'avènement du cinéma-attraction à la fin du XIX ${ }^{\mathrm{e}}$ siècle jusqu'à la sortie du film de Walt Disney Mélodie du Sud en 1946, les films d'animation américains ont représenté les individus noirs de façon stéréotypée. Dépeints comme des voleurs, des tricheurs idiots, dévots à l'extrême ou au contraire blasphémateurs, prompts à la superstition et irrémédiablement esclaves de leurs passions et des plaisirs charnels, les personnages noirs du cinéma d'animation sont les héritiers d'une longue «tradition » visuelle et idéologique antérieure à l'existence du médium lui-même. C'est précisément l'aspect protéiforme de ces représentations et ce qu'elles révèlent des États-Unis qui nous intéresse ici.

From the birth of the cinema-attraction at the end of the 19th century to the public release of the Disney's controversial animated feature-length Song of the South in 1946, the American animated film industry relentlessly depicted Black characters in a stereotypical way. Represented as 
thieves, stupid cheaters, ridiculously religious or on the contrary, blasphemers, superstitious and hopelessly slaves to their passions and carnal pleasure, the animated black characters are as a matter of fact the heirs of a long and deeply-rooted visual and ideological tradition that predates the animation medium itself. I will precisely focus on the multi-layered aspect of such representations and their revealing dimension regarding the history of race in United States.

\section{INDEX}

Mots-clés : cinéma d'animation, racisme scientifique, intermédialité, altérisation, physiognomonie, phrénologie

Keywords : animated films, scientific racism, intermediality, alterization, physiognomy, phrenology

\section{AUTEUR \\ PIERRE CRAS}

Pierre Cras est docteur en civilisation américaine et maître de conférences à l'université Paris 1 Panthéon-Sorbonne. Il est spécialiste d'histoire culturelle et de cinéma et a travaillé pendant sa thèse sur les représentations noires caricaturales dans le dessin animé américain $\mathrm{du} \mathrm{xx}^{\mathrm{e}}$ siècle.

Pierre Cras travaille actuellement sur le cinéma d'horreur comme reflet des angoisses collectives aux États-Unis.

pierre.cras@hotmail.fr 